\title{
Les coparentalités entre gays et lesbiennes en France: le point de vue des pères
}

\author{
Flávio Luiz Tarnovski \\ Universidade Federal de Mato Grosso
}

\section{Resumo}

As famílias constituídas por casais do mesmo sexo se tornaram um importante tema do debate social, político e intelectual francês. Neste artigo, argumento que as famílias homoparentais expressam tendências históricas que transformaram de maneira significativa a organização das relações familiares na França. A partir de uma pesquisa com pais homossexuais franceses, este artigo analisa as especificidades das coparentalidades entre gays e lésbicas. A coparentalidade consiste em um acordo para a geração de crianças, que serão criadas em alternância pelo (s) pai (s) e pela (s) mãe (s). Após analisar as particularidades do projeto parental, dos contornos da família e da experiência da paternidade, proponho uma breve discussão sobre os limites de um modelo exclusivo de filiação.

Palavras-chave: coparentalidade, homoparentalidade, homossexualidade, paternidade, família

\section{Abstract}

The families formed by same-sex couples have become an important topic of the social, political and intellectual debate in France. In this article, I argue that same-sex families express historical trends that have significantly changed the organization of family relations in France. From a survey of French homosexual fathers, this article analyzes the specificities of gay and lesbian co-parenting, which consists in agreements for the generation of children that will be raised separately by the father (s) and the mother (s). After the analyses of the particularities of the parental project, the contours of the family and the experience of fatherhood, I propose a brief discussion about the limits of an exclusive filiation model.

Keywords: co-parenting; gay parenting; homosexuality; fatherhood; family 


\section{Resumé}

Les familles composées par des couples de même sexe sont devenues un thème important dans le débat social, politique et intellectuel français. Dans cet article, je suggère que les familles homoparentales expriment des tendances historiques qui ont significativement transformé l'organisation des relations familiales en France. A partir d'une enquête auprès de pères homosexuels français, ce texte analyse les spécificités des coparentalités entre gays et lesbiennes. La coparentalité consiste en un accord pour la conception d'enfants qui seront élevés en alternance par le(s) père(s) et la(les) mère(s). Après avoir analysé les particularités du projet parental, des contours de la famille et de l'expérience de la paternité, je propose une brève discussion sur les limites d'un modèle exclusif de filiation.

Mots-clés coparentalité; homoparentalité; homosexualité; paternité; famille 


\title{
Les coparentalités entre gays et lesbiennes en France: le point de vue des pères ${ }^{1}$
}

\author{
Flávio Luiz Tarnovski \\ Universidade Federal de Mato Grosso
}

La plupart des sociétés occidentales assistent depuis une quarantaine d'années à des changements importants dans le domaine de la famille et de la conjugalité. Avec la popularisation des méthodes contraceptives, par exemple, la sexualité s'est trouvée dissociée de la procréation (Bozon 2002). Lintervention médicale dans le domaine de la procréation a pour sa part rendue possible la fécondation sans sexualité. L'enfant est de plus en plus le résultat d'un désir et d'un projet qui témoignent de son importance dans la vie du couple, dans la mesure où il devient un "support identitaire " pour des femmes et des hommes voulant être mères et pères (Fine 2001). Dans le contexte actuel, la naissance du premier enfant marque souvent la consolidation du couple hétérosexuel en tant que famille, le mariage n'étant plus une étape obligée dans les trajectoires familiales. On se marie moins souvent et on divorce beaucoup plus. Avec les recompositions qui peuvent suivre les séparations, les enfants sont amenés à circuler dans des réseaux familiaux et voient le nombre d'adultes s'occupant d'eux augmenter. Parallèlement, des changements dans les lois touchant au domaine du couple et de la famille ont, à partir des années 1970, ouvert la voie à une réorganisation profonde de l'univers de liens familiaux. En France, l'instauration du divorce par consentement mutuel, la transformation de l'ancienne puissance paternelle en autorité parentale, ainsi que la suppression des inégalités associées à la filiation, selon qu'elle était légitime ou

\footnotetext{
1 Ce texte reprend de manière synthétique des analyses développées dans ma thèse de doctorat en anthropologie sociale (Tarnovski 2010), soutenue à l'École des hautes études en sciences sociales, sous la direction d'Agnès Fine. Le terrain, mené à Paris et à Toulouse, est constitué d'observations réalisées dans des réunions de pères homosexuels membres de l'Association de Parents et futurs Parents gays et lesbiens/A.P.G.L. et d'entretiens enregistrés avec 23 pères. Cette recherche a été financée par une bourse de la Capes/Ministère de l'Education brésilien.
} 
naturelle, ont redessiné les contours de la famille, qui devient multiple dans ses manifestations (voir Théry 1998). Après les familles monoparentales et recomposées ou encore celles ayant fait appel à l'aide médicale à la procréation, les familles homoparentales sont passées sur le devant de la scène, concentrant autour d'elles des inquiétudes en rapport avec la filiation, la parentalité ou la différence des sexes (Théry 1996; Ouellette 2000).

En France, c'est au cours des années 1990 que les parents gays et lesbiens deviennent visibles. ${ }^{2}$ Même si leur nombre n'est pas important d'un point de vue statistique (Festy 2006), la demande de reconnaissance juridique des familles homoparentales interroge les principes et les valeurs qu'une société se donne comme références pour l'inscription officielle des liens de parenté. D’une manière générale, les familles homoparentales posent la question du lien entre sexualité et procréation, conjugalité et parentalité et entre alliance et filiation (Cadoret 2002). Elles ne sont pas les seules, parmi les configurations familiales contemporaines, à produire des dissociations entre ces différentes dimensions, mais c'est dans leur cas que les enjeux contemporains liés à la création des liens de parenté deviennent les plus évidents. Ne pouvant pas superposer ces dimensions de façon à correspondre à l'idéal de leur société, dont la réalisation concrète dans le passé semble parfois surestimée, ces différentes configurations homoparentales mettent en lumière les principes qui structurent et organisent l'univers des relations familiales et des liens de parenté dans les sociétés occidentales contemporaines. Si, en ce sens, l'action de l'Association des Parents et futurs Parents gays et lesbiens (A.P.G.L.) a contribué à fédérer les demandes de pères et mères homosexuels sous la bannière de l'homoparentalité, il n'en reste pas moins que cette notion fait référence à des configurations familiales très diverses. ${ }^{3}$

La plus classique d'entre elles est constituée par des hommes ou des femmes ayant eu leurs enfants dans le cadre d'une union hétérosexuelle et qui, après la rupture de cette union, ce sont assumés comme homosexuel(le) s. L'on peut y trouver des trajectoires et des attitudes face à la sexualité et à la

2 Pour des questions de style, j'utiliserai indistinctement les catégories homosexuel, homosexuelle, gay et lesbienne. Ces catégories ne sont pas forcément celles utilisées par les sujets enquêtés eux-mêmes pour s'auto-désigner, qui peuvent préférer celles d' " homo " ou de " pédé ». Néanmoins, l'utilisation des termes supra est largement diffusée dans la population étudiée. Pour une analyse des dimensions sociales et historiques de ces catégories voir Foucault (1994).

3 La notion d'homoparentalité a été créée par l'A.P.G.L. pour désigner les familles dont l'un des parents au moins s'assume comme homosexuel (Gross 2005). 
parentalité très contrastées. Si bien que les recompositions familiales homoparentales ne sont probablement pas vouées à disparaître, elles témoignent d'une époque où l'accès à la paternité et à la maternité était très restreint dans le cas d'individus s'assumant comme homosexuels. En France, cet horizon s'est élargi considérablement au cours des années 1990 et d'autres modes d'accès à la parentalité ont vu le jour. Un nouveau discours social sur l'homosexualité commence à se construire proposant une conjugaison positive entre homosexualité et paternité/maternité. S'assumer comme gay ou lesbienne n’impliquerait plus nécessairement un "renoncement" au désir d'avoir un (des) enfant (s) ou de fonder une famille avec un partenaire de même sexe. Ainsi, de plus en plus de gays et de lesbiennes se lancent dans des procédures d'adoption, la loi française ne l'interdisant pas, en principe, à des candidats célibataires. D'autres font appel à l'aide médicale à la procréation, que ce soit par don de sperme dans le cas des femmes, ou du recours à une mère porteuse (avec ou sans l'utilisation d'un don d'ovocytes) pour les hommes, mais à l'étranger, car la France n'autorise pas l'accès à ces techniques aux couples de même sexe. À titre de comparaison, au Brésil certains homosexuels font appel à une sorte d'adoption informelle, à travers laquelle un enfant donné par une femme, trouvée grâce à des réseaux d'interconnaissance, est reconnu comme étant sien devant l'état civil par le père " adoptif " (Tarnovski 2002). Le contraste avec les pratiques des pères homosexuels brésiliens montre qu'en France la loi n'est pas contournée de la même manière... Dans le cas de la G.P.A. (Gestation pour Autrui), comme elle est illégale sur le territoire français, les homosexuels qui veulent y avoir recours se déplacent vers des pays où elle est légalisée. En ce sens, même si leur situation familiale ne peut pas être reconnue par le système juridique français actuel, leur famille ne s'est pas constituée illégalement. Enfin, une quatrième possibilité de fonder une famille lorsque l'on est gay ou lesbienne consiste à réaliser des coparentalités, c'est-à-dire, s'associer avec un partenaire de l'autre sexe, selon des modalités très variées, pour faire un enfant, avec ou sans rapports sexuels, et éventuellement l'élever ensemble.

La coparentalité, objet de cet article, est le résultat de la convergence de tendances à l'œuvre dans la contemporanéité. La valorisation du désir d'enfant, du projet de famille, de l'authenticité de relations fondées sur le choix, de la réalité des soins prodigués au quotidien, de l'affection, sont autant de critères définissant la famille contemporaine et qui trouvent leur expression 
dans ces configurations familiales (Théry 1998; Fine 2001; De Singly 2004; Godelier 2004). Si, d'une part, les configurations coparentales peuvent être considérées comme les plus proches des formes familiales connues, vu que l'enfant a un père et une mère, de l'autre, elles posent la question de la pluriparentalité (Fine 2001). La « différence des sexes », notion centrale dans le débat français sur l'homoparentalité, n'est pas " effacée ", mais les liens de parenté se multiplient au-delà du modèle d'exclusivité selon lequel chacun n'a qu'un père et qu'une mère (Ouellette 1998; Cadoret 2002; Fassin 2005). Quelles sont les implications de ces faits sur l'organisation du quotidien familial ? Comment les tendances contemporaines dans le domaine du couple et de la famille affectent-ils ces formes familiales ? Considérant la nouveauté de ces montages familiaux, comment les parents réactualisent-ils les représentations sur la parenté ? À partir de l'analyse de certains aspects de la construction du projet de coparentalité et des enjeux liés à sa réalisation, cet article proposera des éléments de réponse à ces questions.

\section{Le projet de coparentalité : construire la parentalité avant l'arrivée de l'enfant}

Fondée en 1986, l'Association des Parents et futurs Parents gays et lesbiens s'est, dès le début, constituée en espace d'échange " convivial » entre parents homosexuels. Sa création s'inscrit dans un contexte historique singulier concernant la structuration du mouvement homosexuel français, qui s'oriente progressivement vers d'autres centres d'intérêt que la seule lutte politique. Comme le montre Peerbaye, de nouvelles associations ont vu le jour ayant pour caractéristique principale " [...] d'être des groupes d'individus homosexuels partageant en outre une seconde qualité (un loisir, une appartenance religieuse, l'appartenance à une classe d'âge...), et désireux de se rassembler sur la base de cette "communauté d'appartenance" " (Peerbaye 2000: 45). Ne comptant qu'une centaine de membres dans les premières années de son existence, l'Association permettait à des pères et mères qui voulaient s'assumer comme homosexuels d'échanger sur des thèmes communs. Comme il leur donnait le sentiment de n'être plus les seuls dans cette situation, le fait de se retrouver " entre soi » leur a permis de conjuguer positivement parentalité et homosexualité. Aujourd'hui, l'A.P.G.L. compte plus de 1600 adhérents, répartis entre différentes configurations familiales, créées 
à partir de l'adoption, de la coparentalité, de l'insémination avec donneur ou de la gestation pour autrui. D’après les statistiques fournies par l'A.P.G.L., l'Association comptait en 1998, 255 hommes (35\%) et 473 femmes (65\%), en 2002, 401 hommes (28\%) et 1033 femmes ( $72 \%)$ et en 2006,458 hommes $(28 \%)$ et 1153 femmes ( $72 \%)$.

Selon Martine Gross, « la très grande majorité des hommes (85\%) de l'A.P.G.L. qui souhaitent devenir parents se tournent [...] vers la coparentalité, alors que seules $40 \%$ des femmes choisissent cette configuration familiale " (Gross 2006: 153). Cette asymétrie entre hommes et femmes s'expliquerait par la facilité d'accès qu'ont les femmes à des techniques de procréation médicalement assistée. Quoique interdites en France, lorsqu'il s'agit d'une femme célibataire ou en couple avec une autre femme, certaines techniques comme l'I.A.D. (Insémination avec Donneur) sont autorisées dans des pays voisins, comme la Belgique et l'Espagne et celles qui veulent avoir recours à un donneur connu peuvent se rendre aux Pays Bas. ${ }^{4}$ L'assistance médicale à la procréation ne peut pas encore répondre au désir masculin d'enfant avec la même facilité. La gestation pour autrui, outre le fait d'être beaucoup plus coûteuse que l'I.A.D., demande le concours d'une femme prête à porter l'enfant et à renoncer aux droits qu'elle aurait sur lui, tandis que pour une I.A.D. il suffit qu'un homme soit disposé à donner son sperme. En ce qui concerne l'adoption, comme solution pour accéder à la parentalité, Gross souligne la résistance encore persistante des services sociaux à donner un agrément pour l'adoption à des hommes célibataires, décourageant ainsi une partie de ceux qui l'envisageait comme possibilité d'accès à la paternité. De sorte que la coparentalité serait, pour des homosexuels voulant devenir pères, le moyen le plus accessible.

Parallèlement, dans le débat public sur l'homoparentalité, les opposants font appel à des théories psychologiques pour affirmer qu'un enfant aurait besoin d'un père et d'une mère pour s'épanouir (Mehl 2003). Les gays et les lesbiennes voulant fonder une famille se sentent souvent dans l'obligation de

4 Dans ces pays, outre le fait qu'il n'est pas interdit, l'accès à l'insémination avec donneur anonyme est encadré par des équipes interdisciplinaires devant lesquelles les lesbiennes françaises n'ont pas besoin de cacher leur vie de couple, bien au contraire. Dans certains hôpitaux belges, il y aurait même une préférence pour des femmes en couple, plutôt que pour des femmes célibataires. Certaines femmes s'étonnent de cette réceptivité, habituées qu'elles étaient à l'image de transgression associée à ce type de projet en France (Descoutures 2008: 131). 
répondre à ces normes sociales. Dans ce contexte de prolifération de discours savants autours de la parentalité, le choix de la coparentalité peut être déculpabilisant dans la mesure où l'enfant aura un père et une mère (Gross 2006).

La coparentalité permet également d'avoir un enfant « de sa propre chair ", pour reprendre ici l'expression employée par certains pères interviewés. André, 531 ans, enseignant, était disposé à envisager l'adoption pour un deuxième enfant, mais seulement après en avoir engendré un premier. Ici le désir d'enfant est dissocié, pour ainsi dire, du désir de paternité, ce dernier ne pouvant se réaliser complètement qu'à travers l'engendrement. ${ }^{6}$ Par ailleurs, la dynamique d'alternance des foyers propre à la coparentalité peut également être perçue comme un avantage. Comme l'affirme Bernard, 37 ans, orthophoniste,

[...] je lui trouve un intérêt superbe [à la coparentalité], c'est de pouvoir continuer à vivre ma vie de couple, sans enfant, avec Bruno, avec tout ce que ça implique de sorties, de balades, et de vivre ce désir d'enfant également en accueillant l'enfant et en vivant là complètement l'implication avec lui (Bernard).

Ainsi, parallèlement à l'argument selon lequel la coparentalité répondrait à une préoccupation de donner un père et une mère à l'enfant, que ce soit pour reproduire un certain modèle de normalité familiale ou en réaction au débat public sur les familles homoparentales, la garde partagée de l'enfant est considérée par certains pères comme un aspect positif des arrangements coparentaux. Cela semble indiquer que, en contraste avec l'idée de ce que les pères gays se trouveraient « soumis » à un modèle de famille caractérisé par la prévalence maternelle, tous ne veulent pas s' " affranchir " de ce modèle, au moins pas complètement.

Dans mon enquête, parmi les quinze pères ayant opté pour une coparentalité, cinq ont connu la mère de leurs enfants grâce à leur réseau d'amis, trois par des annonces et sept lors de "réunions conviviales " organisées par des membres de l'A.P.G.L.. Le choix de la future mère passe par un certain nombre de critères, très variables, plus ou moins négociables, mais dans certains cas une forme de séduction n'a pas été étrangère à ce processus. Bernard

5 Les prénoms ont été changés pour préserver l'anonymat de mes interlocuteurs.

6 Chez les femmes, ces différents contenus du désir peuvent se traduire par la distinction entre « désir d'enfant » et " désir de grossesse " (voir Descoutures 2008). 
raconte comment lui et son compagnon, Bruno, 45 ans, médecin, ont rencontré les femmes avec lesquelles ils recherchaient une coparentalité:

[...] il est indéniable qu'il y a un côté séduction volontaire et conscient, voilà. Et donc il y avait notamment... alors, moi j’ai senti que ça pouvait être possible mais qu'il fallait qu'on se rende attrayant. [...] J'ai proposé à Bruno de danser avec moi et nous avons dansé amoureusement et puis je gardais un œil sur les filles, qui nous regardaient et qui avaient l'air de se dire « mais qu'ils ont l'air trop mignon tous les deux; qu'est-ce que ce serait mignon... " (rires), " Ah, et comme ils s'occupent bien des enfants des autres... ", il y avait un côté un peu comme ça. Et puis alors, quand [la future mère] nous en parle, elle nous le dit rétrospectivement : « Vous aviez l'air amoureux, vous étiez bien avec les enfants et tout ça ». Moi je rigolais intérieurement parce que je savais qu'il y avait ce côté un petit peu, comment ?... On était en train de se vendre quoi. Mais comme une étape de séduction dans un couple, si tu veux, où... (pause) comme, comme quand tu essaies de séduire un partenaire, mais avec lequel tu vas avoir une relation sexuelle, mais tu séduis un partenaire dans la perspective d'avoir un enfant mais avec d'autres, d'autres paramètres du coup (Bernard).

La scène décrite se passe lors d'une réunion conviviale entre membres d'une antenne régionale de l'A.P.G.L.. Bernard raconte également que, après les premiers contacts avec le couple de femmes pendant ces réunions, son compagnon Bruno a rencontré la future mère dans d'autres situations, de façon imprévue, « et donc ils se sont dit que ça les travaillait fort cette histoire-là ». Ces rencontres fortuites les auraient encouragées à poursuivre les conversations sur un possible projet de coparentalité. Les couples se sont fréquentés par la suite, afin de discuter des aspects pratiques liés à la mise en place de leur projet commun.

La référence à la « séduction " comme critère de sélection d'une mère potentielle lors de rencontres conviviales organisées par l'A.P.G.L., a été aussi mise en avant par Jean, 43 ans, ingénieur. Il raconte avoir eu un " petit coup de foudre " pour la future mère de sa fille et déclare qu'il l'a trouvée physiquement attrayante : " [...] J'ai dit tiens, elle est vraiment pas mal celle-là. » Cette logique de séduction semble être inhérente à la dynamique des réunions conviviales entre gays et lesbiennes en quête de coparents, car, contrairement à ce qui se passe lors des rencontres en face-à-face, en réponse à une annonce, par exemple, il faut que les futurs coparents puissent se choisir 
selon des critères qui ne soient pas uniquement ou explicitement objectifs. Autrement dit, les réunions conviviales mettent en relation plusieurs coparents potentiels qui doivent trouver des critères non explicitement objectifs ou pragmatiques sur lesquels fonder leur choix, ce qui expliquerait l'entrée en jeu d'une logique de séduction.

Dans certains cas, mes interlocuteurs ont dû rencontrer plus d'une femme ou d'un couple de femmes avant de choisir la future mère de leurs enfants. Parmi les critères énoncés pour choisir une partenaire, il y a d'un côté tout l'aspect pratique lié à la gestion de la coparentalité, c'est-à-dire la résidence de l'enfant, le nom de famille, 7 la place des partenaires, à propos desquels il faut se mettre d'accord, mais aussi des critères plus subjectifs liés à des définitions de la masculinité et de la féminité, à l'apparence physique, ainsi qu'au capital scolaire et culturel. Ainsi, un idéal d' « homogamie » semble orienter cette recherche de partenaires en vue d'une association pour faire ensemble un ou plusieurs enfants et l'(les) élever en alternance.

Tous ne sont pas d'accord sur le cadre éthique qui devrait orienter cette " sélection ", certains ayant exprimé leur malaise face aux « castings " réalisés par certains futurs parents. Cela semble indiquer que, "vidées " de la conjugalité, les unions réalisées dans ce contexte ne semblent pas pour autant exemptes d'une dimension affective, en quelque sorte intangible, liée à l'idée de " rencontre ", sur laquelle les acteurs cherchent à faire reposer le lien qui les unit par-delà le seul projet parental. Dans cet esprit, il peut y avoir toute une période pendant laquelle ils sortent pour dîner ensemble et font la connaissance des amis respectifs pour mieux se connaître et trouver des " affinités ", période qui peut s'étendre de quelques mois à un an ou plus. Certains hommes en coparentalité avec des amies de longue date confirment, mais à l'inverse, l'importance de construire un lien avec la future mère, lorsqu'ils disent qu'ils n'auraient peut-être jamais fait une coparentalité avec une " inconnue " si leur amie ne leur avait pas donné l'occasion d'être pères. La connaissance des familles respectives, contrairement à celle des amis, ne

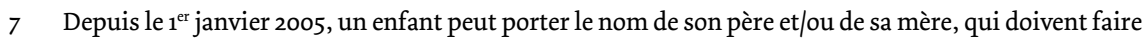
une déclaration conjointe de choix du nom. Les coparents homosexuels étant dans la même situation que les couples en union libre, en absence de cette déclaration, c'est l'ordre d'établissement des filiations qui déterminera le nom de l'enfant. Celui-ci portera le nom du parent qui l'a reconnu en premier. Lorsque la reconnaissance est faite conjointement par le père et par la mère, l'enfant portera le nom du premier si aucune déclaration conjointe de choix de nom n'est déposée par les parents. 
semble pas être une préoccupation centrale pendant cette phase. D’ailleurs, d'une manière récurrente les pères interviewés ont dit n'avoir " informé " leur famille de ce projet parental que dans l'imminence de l'arrivée des enfants.

La particularité du projet de coparentalité réside dans le fait qu'un nombre important d'aspects futurs liés au quotidien de la parentalité sont ou " devraient être " discutés avant la conception de l'enfant. Cette anticipation des rôles et de la manière dont se concrétisera le quotidien familial se retrouve aussi lorsqu'il s'agit d'un projet d'adoption ou de G.P.A.. Dans cet esprit, un couple qui allait bientôt accueillir un enfant adoptif m'a expliqué quel allait être le rôle de chacun, comment ils se feraient appeler par l'enfant, etc.... La différence étant que les acteurs de la coparentalité auront une marge d'adaptabilité moins importante, une fois l'enfant arrivé, par rapport à ce qui peut se passer dans les autres configurations, spécificité liée à la dissociation entre conjugalité et parentalité. Le projet, dans les coparentalités, ne concerne pas seulement la réflexion sur les possibilités concrètes de réalisation d'un désir d'enfant et la planification de son arrivée, mais également la prévision de la forme et du contenu, autant que faire se peut, du rôle de chaque coparent. Si la période de fréquentation entre les futurs coparents permet de construire un lien en amont de l'engendrement, elle n'est pas moins importante, selon eux, vis-à-vis de la mise au clair du projet de coparentalité, c'est-à-dire de l'ensemble d'aspects pratiques et subjectifs liés à l'exercice de la parentalité.

A défaut d'être liés par des liens d'amitié remontant à plusieurs années, et malgré l'existence d'une période de fréquentation initiale, le lien entre les coparents trouve appui sur l'accord ou le « contrat " qui précède l'engendrement, qu'il soit explicite ou implicite, écrit ou seulement oral, dans la mesure où il les oblige l'(les) un(s) envers l'(les) autre(s) et modèle leurs actions en tant que parents, tout comme leur relation en tant que coparents. Le nonrespect de cet arrangement impliquant la rupture de l'accord, il peut exiger un règlement judiciaire. Comme l'A.P.G.L. a bien compris les enjeux de la coparentalité, et notamment l'importance de la phase d'élaboration du projet, elle propose des modèles de charte pour orienter les futurs coparents dans leur négociation (Gross \& Peyceré 2005). Mais la mise en place d'une charte n'est pas toujours simple. Après s'être vu refuser l'agrément d'adoption, Eugène, 44 ans, enseignant, a commencé à rencontrer des femmes à 
la recherche d'une coparentalité. Le premier couple rencontré ne voulant pas céder sur certains points du projet, Eugène s'est lancé à la recherche de femmes plus à même de répondre à ses attentes. Selon lui, « on avait l'impression d'inventer quelque chose à cette époque-là (en 1995). » Un accord a fini par être trouvé avec un deuxième couple et l'enfant a été conçu quelques rencontres plus tard. Toutefois, pendant la gestation, Eugène est tombé sur un article du journal interne de l'A.P.G.L., écrit par un « papa qui s'est fait avoir par la maman ". Selon lui, l'auteur de ce texte s'adressait à des hommes voulant faire une coparentalité pour leur recommander vivement de faire une reconnaissance anténatale et de valider un accord devant le juge. Cette prise de conscience soudaine de la fragilité de sa position lui a fait demander à la future mère d'aller reconnaître l'enfant avec lui.

Alors que je m'entendais très bien avec la future maman, j'ai lui ai dit : « Tu sais, on va aller reconnaître l'enfant », elle était enceinte, elle l'a tout de suite mal pris. Et je lui ai dit : « et après on passera devant le juge, on mettra tout [sur le papier] ", et vraiment elle l'a mal pris, je ne comprenais pas pourquoi. « Mais attends, ce n'est rien ", "Ça y est, tu me soupçonnes », " Je ne te soupçonne de rien du tout ». Et c'était très tendu quand on est allé chez le juge (Eugène).

L'importance qu'a l'accord ou l'engagement mutuel entre les futurs coparents, soulignée par la plupart des enquêtés dans cette situation, contraste avec la difficulté ressentie par certains de pouvoir parler avec sérénité des aspects juridiques, organisationnels et quotidiens du projet une fois la coparentalité établie.

C'est ainsi que, Fabien, 50 ans, chef d'entreprise, dans une toute autre optique, n’a voulu signer aucun papier avec la mère de ses deux enfants. Lorsqu'ils se sont rencontrés, aucun des deux ne connaissant l'existence de l'A.P.G.L., leur projet a été élaboré à l'écart de l'expérience que commençait à accumuler l'Association. Au lieu de signer une charte, les deux futurs coparents ont fondé leur accord sur la " confiance ". Même si cette confiance en l'autre était également présente dans les autres situations enquêtées, ce qui semble particulier chez Fabien, c'est le refus de la trace écrite comme support de leur accord.

Notre accord, ça a été droit dans les yeux [...] La base de notre accord c'est : « on aura des différends, on aura des choses, mais pas de conflits. S’il y a conflit on 
résout. " Ça a été vraiment la base. [...] Les chartes, tout ça c'est très bon, pourquoi pas, mais enfin ça amène beaucoup d'ambiguïtés aussi, parce que dans une relation, c'est pas parce qu'on a écrit sur un papier que... il y a des gens qui sont mariés, ils divorcent, ils s'entretuent, hein ! et ils ont signé. [...] J’ai refusé de... je n'ai pas voulu... " on se parle, moi je te crois ". Si on ne rentre pas dans une relation de ce type, de croyance en l'autre, on va être... on va se faire des procès parce qu'on a un papier qui... ça n'a pas de sens. Les enfants n'ont pas besoin de ça (Fabien).

Pour lui, la parole est plus importante qu'un texte écrit. Le fait que leur partenaire conjugal n'ait pas fait partie du projet initial n'est pas à exclure comme condition pour qu'un tel engagement puisse fonctionner dans la durée. De même, Jean ne voulait pas non plus signer de charte de coparentalité, mais dans son cas elle a été rédigée. Lui et le couple de femmes avec lequel il allait avoir un enfant se sont basés sur un modèle proposé par l'A.P.G.L. afin de clarifier leur vision de la coparentalité. Une fois l'accord trouvé, ils ont déchiré la charte qu'ils venaient pourtant de mettre au point. Selon Jean, " Ça n'a vraiment pas de sens de signer un papier à la naissance, il n'est pas valable toute la vie, il change sans arrêt. Donc l'on s'est dit : "on se fait confiance" et puis voilà. Et ça marche d'ailleurs très bien. " Dans certains cas, l'ombre du divorce, dans sa dimension conflictuelle et procédurale, peut rendre peu aisée l'explicitation de la vision de chaque couple ou chaque individu sur la coparentalité. Mais à la différence des couples mariés confrontés à un divorce, dans les coparentalités, un compromis entre les coparents doit être trouvé alors que l'enfant n'est même pas encore né.

\section{Des familles aux contours variables}

Les configurations familiales créées par les arrangements coparentaux entre gays et lesbiennes sont celles qui semblent se distancier le moins des formes familiales connues. A première vue, leur situation ressemble à celle des familles recomposées. Anne Cadoret (2002) remarque que, malgré cette apparence de similitude, les coparentalités présentent une différence importante : elles ne sont pas issues d'une recomposition, mais d'une composition. Autrement dit, dans les coparentalités, le père et la mère n'ont jamais constitué un couple et l'enfant est dès sa naissance dans un contexte de pluriparentalité (Fine 2001). Contrairement aux familles recomposées, les 
" constellations familiales " créées par la coparentalité ne sont pas « héritières " d'unions antérieures (Théry 1991). Les coparentalités reprennent cependant l'image d'un réseau de familles, habituellement associée à la recomposition après divorce, entre lesquelles circule l'enfant. Dans les coparentalités, cette ouverture vers l'extérieur étant constitutive, les enchaînements successifs entre couples d'hommes et de femmes peuvent aussi, quoiqu'un peu différemment, générer une organisation des familles en réseau. Comme la notion de réseau de familles laisse penser qu'il y a des liens actifs entre les différentes unités, cette image de familles en réseau semble plus appropriée pour décrire le type de connexions établies par la coparentalité. Selon l'un des mes interlocuteurs, il s'agirait de «familles qui s'emboîtent dans tous les sens ». Le diagramme 1 illustre la forme que peuvent prendre ces enchaînements.

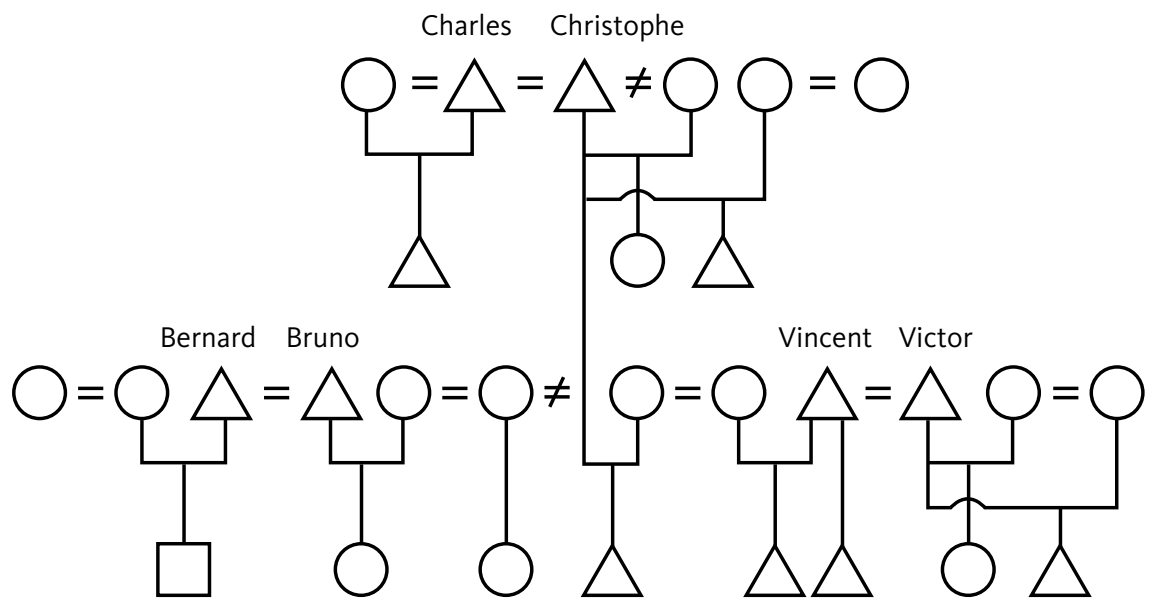

Diagramme 1: Enchaînement de coparentalités

Les traits verticaux représentent les liens de filiation officiellement reconnus. Les liens de filiation dits " sociaux ", eux, demeurent plus difficiles à dessiner, car ils varient selon les définitions qu'en donnent les différents acteurs de la famille. Une première différence, par rapport aux enchaînements produits par les familles recomposées, apparaît dans le rapport entre alliance et filiation. Dans la coparentalité, ces deux dimensions de la parenté ne se superposent pas complètement, ce qui constitue une marque structurelle de ces configurations familiales. Le père et la mère légaux n'ont jamais été en 
couple, de sorte que les liens de filiation à leur égard n'ont jamais trouvé leur justification dans une relation d'alliance. Celle-ci, lorsqu'elle existe, s'établit entre les partenaires de même sexe. Le lien entre le père et la mère biologiques et légaux prend ainsi la forme d'un lien d'affinité qui passe par l'enfant.

D’une manière générale, dans les situations enquêtées, les rapports avec la famille de l'autre parent ne sont pas fréquents, et peuvent même être très limités. Parfois, ils ne font connaissance qu'au moment de la naissance, à la maternité. Certes, de nos jours, ce contact entre familles n'est plus aussi régulier ni aussi important qu'il a pu l'être de par le passé. Mais à la différence de la situation des couples hétérosexuels, les hommes et les femmes en coparentalité n'ont d'autre lien entre eux que celui découlant du fait d'avoir engendré et reconnu un enfant ensemble. Ils demeurent, en quelque sorte, des " étrangers " pour la famille de l'autre parent.

Le rapport entre les familles respectives des parents légaux dépend dans une large mesure de la qualité et du type de lien entretenu par ces derniers. Dans les situations de conflit, les contacts perdent toute justification, se limitant à une " cohabitation " temporaire lors des fêtes d'anniversaire de l'enfant, par exemple. Au-delà de la qualité des relations entre coparents, le rapport qu'ils entretiennent entre eux, et avec les familles respectives, dépend également de leur vision de la coparentalité, notamment de la définition qu'ils donnent à la famille. Certains dessinent les contours de la famille à partir de l'enfant, en y incluant l'(les) autre(s) parent(s), alors que d'autres partent de la réalité conjugale et excluent les partenaires coparentaux de leur définition de la famille.

Emmanuel Gratton a identifié trois modèles distincts pouvant orienter les acteurs de la coparentalité dans leurs rapports, qu'il nomme les modèles exclusif, bipolaire et intégratif (Gratton 2008: 208). Dans le premier, les géniteurs se considèrent comme les seuls parents de l'enfant, la conjugalité étant ici nettement dissociée de la parentalité. Ce modèle serait à l'œuvre surtout dans les situations où les partenaires de la coparentalité ne vivent pas en couple ou quand les partenaires conjugaux n'ont pas participé à l'élaboration du projet parental. L'auteur considère que ce modèle exclusif est plus fréquent chez les hommes que chez les femmes, pouvant entraîner une dissymétrie entre les partenaires de la coparentalité. Dans le modèle bipolaire, l'accent mis sur la conjugalité opère une distinction entre deux unités familiales distinctes. Les différences à l'intérieur du couple seraient moins marquées, 
mais plus visibles dans l'opposition entre les deux pôles représentés par le couple d'hommes et le couple de femmes. Ainsi, dans ce modèle, il y aurait une tendance à l'intégration de la famille du compagnon ou de la compagne. Selon l'auteur, «le modèle bipolaire, qui reconnaît la "différence des sexes" par la "différence des pôles", insiste donc moins sur la "différence des rôles" au sein du couple conjugal. » Et il ajoute : « Le "domestique" prime ici sur le "biologique". " (Gratton 2008: 219) Enfin, le modèle intégratif serait plus axé sur un sentiment de coopération parentale et d'unité familiale autour des deux couples. Selon l'auteur, plus difficile à mettre en œuvre, ce dernier exigerait des "principes communautaires ", difficilement conciliables avec les revendications particulières de chaque parent ou couple. Les coparentalités auraient donc tendance à osciller entre le modèle intégratif et le modèle bipolaire. Tout cela pouvant changer avec le temps, la forme des arrangements coparentaux dépendrait de confirmations permanentes de la part des acteurs.

Ces différentes logiques d'articulation entre procréation, parentalité et conjugalité ont été retrouvées dans mon terrain. Pour la naissance de son premier enfant, Fabien et Françoise ont réalisé une coparentalité " à deux ", sans la participation d'éventuels compagnon ou compagne. Après la naissance, Fabien s'est installé chez Françoise afin de l'aider avec le bébé et de rester près de lui. Après quelques mois, il a commencé à prendre l'enfant seul chez lui, mais sans avoir mis en place un cadre strict de visites. Les contacts entre lui et la mère étaient fréquents et ne suivaient pas une routine prédéterminée. Cela correspondait à un souhait de sa part de construire un sentiment d' " unité familiale ». Rétrospectivement, Fabien caractérise cette phase comme une période de " vies entremêlées ". A un moment donné, juste avant la naissance de leur deuxième enfant, ils ont limité leurs rapports et instauré une distinction plus claire entre les sphères de la parentalité et de la conjugalité. Fabien a notamment soutenu la participation de la compagne de Françoise dans le projet d'un deuxième enfant. De son côté, il s'est engagé dans une relation de couple, mais sans pour autant vouloir inclure son compagnon dans ce projet. La caractérisation des premières années par la notion quelque peu négative de " vies entremêlées » révèle les limites de l'idée d' " unité familiale ».

Cet exemple nous montre également que le point d'équilibre de la coparentalité n'est pas exactement le même que celui de la recomposition après divorce. Si dans les familles recomposées l'équilibre consisterait à maintenir des relations amicales entre parents malgré la séparation du couple, dans les 
coparentalités, au contraire, il s'agirait plutôt d'éviter la proximité « excessive », " malgré » la bonne entente, le risque étant que la relation entre parents prenne la place de la relation conjugale. C'est effectivement ce qui s'est passé dans le cas de Robert, 43 ans, publicitaire, son ex-compagnon estimant qu'il n'y avait plus de place pour lui dans son projet de coparentalité. Après la rupture du couple, Robert est allé résider avec la future mère, laissant temporairement en suspens sa vie conjugale. Ce n'est qu'après la naissance de l'enfant qu'un nouvel équilibre entre la parentalité et la conjugalité a été trouvé, avec une séparation plus claire entre le pôle paternel et le pôle maternel et la remise en couple de l'un et de l'autre.

Contrairement à Fabien et à Robert, Vincent, 42 ans, infirmier, n’a pas décrit une phase de rapports privilégiés avec la mère de son fils, mais il n'a pas non plus voulu que les relations entre les couples soient " trop mêlées ". Les vacances passées ensemble, par exemple, même si jugées bénéfiques pour l'enfant qui peut se retrouver en même temps avec tous ses parents, sont aussi considérées comme source potentielle de conflits. Le fait que les deux couples se retrouvent ensemble fait ressortir, par exemple, les différences dans leur manière d'éduquer l'enfant. Si ces dernières ne ressortent pas au quotidien, c'est que l'enfant adapte son comportement en fonction des règles de chaque couple de parents. Lorsque ceux-ci se retrouvent ensemble pendant une période plus longue, comme pendant les vacances, l'enfant doit se référer à des normes hétérogènes, révélant ainsi au grand jour les différences entre les cadres posés par chaque couple.

Les relations entre les " pôles " varient aussi en fonction du nombre d'enfants que veut chaque couple ou individu en coparentalité. Un même couple de femmes, par exemple, peut être en coparentalité avec deux couples d'hommes et vice-versa. L'application des modèles d'articulation entre conjugalité et parentalité identifiés par Gratton devient plus complexe, car différentes logiques peuvent être à l'œuvre dans les rapports entretenus par les différents acteurs des coparentalités. La logique intégrative s’applique difficilement comme modèle prépondérant, mais elle peut être temporairement mise en valeur pendant des occasions spéciales. Jean, par exemple, a réalisé une coparentalité avec une femme dont la compagne avait déjà une fille avec un couple gay. Selon lui, les deux enfants se considèrent comme des « demisœurs ", même si elles n'ont aucun parent « biologique » ou légal en commun. En conformité avec cette définition de la famille, Jean accueille parfois 
les deux petites filles qui sont aussi traitées comme des " demi-sœurs " par les parents de Jean. Comme chacun entretient de bonnes relations avec le père de l'autre enfant, ils ont mis en place un système de relève pour prendre et ramener les enfants chez le couple de femmes et éviter que tous deux aient à se déplacer. Les week-ends à la campagne, chez Jean, sont aussi des occasions où tous les parents peuvent se retrouver ensemble, confirmant ainsi la notion d'une grande famille.

Dans le cas de Vincent, les rapports avec Christophe, 66 ans, enseignant retraité, également en coparentalité avec le même couple de femmes, ne sont pas aussi réguliers. D’une part, Christophe réside à $700 \mathrm{~km}$ de son fils et les occasions pour que les coparents se rencontrent ne sont pas aussi fréquentes, ${ }^{8}$ et de l'autre, lorsque Christophe a réalisé sa coparentalité, la mère de son fils était en couple avec une autre femme. Dans leur cas, la coparentalité s'entrecroise avec une recomposition, ce qui expliquerait les rapports moins réguliers entre Christophe, Vincent et Victor. D'ailleurs, d'après les deux premiers, leurs fils ne se considèrent pas comme des " frères".

Les enchaînements créés par les coparentalités posent ainsi la question du lien de fraternité entre enfants issus de parents distincts mais élevés ensemble au sein d'un même foyer. La situation des enfants de Vincent, Victor et Christophe est emblématique des enjeux liés aux coparentalités entre gays et lesbiennes. On y trouve, d'une part, deux enfants élevés par un même couple de femmes et qui ne se considèrent pas comme frères, et de l'autre, des enfants n'ayant pas la même résidence, ni les mêmes parents légaux, et qui sont amenés à nouer des liens de fraternité, en l'occurrence de quasi-fraternité. C'est le cas des enfants de Vincent et Victor, le premier ayant également adopté un garçon. Ainsi, lors de la naissance de la fille de Victor, 40 ans, infirmier, le père et son compagnon ont envoyé des faire-part où les deux fils de Vincent annonçaient l'arrivée de leur petite sœur. Dans ces situations, le lien fraternel peut-être soit confirmé soit fragilisé selon que les enfants portent ou pas le même nom. Dans le cas de Vincent, le fait que ses deux fils, l'un adoptif et l'autre issu d'une coparentalité, portent son nom, a été mis en avant comme un élément de confirmation du lien de (demi-)fraternité.

Si d'une part, les liens de fraternité dépendent en partie de la définition

8 Généralement, Christophe se déplace pour voir son fils une fois par mois et celui-ci va à Paris chez son père environ quatre fois par an. 
de la famille agencée par chaque " pôle » de la coparentalité, l'arrivée de nouveaux enfants est une décision qui engage tous les coparents. Mon enquête, ainsi que des recherches précédentes (Cadoret 2002; Gratton 2008), semble indiquer que les coparentalités croisées, c'est-à-dire une situation où deux hommes en couple auraient chacun un enfant avec deux femmes en couple, n'est pas une pratique courante. Lorsque la décision d'avoir un deuxième enfant est à l'ordre du jour, soit ce sont les mêmes géniteurs qui s'accordent pour faire un autre enfant, comme cela a été le cas pour Fabien et Françoise, soit c'est le compagnon qui devient père à son tour, à travers une autre coparentalité avec un nouveau couple. La situation de Victor, qui a eu un enfant avec chaque femme d'un couple, et celle de Gabrielle, 43 ans, comptable, qui a fait un enfant avec chacun des deux hommes en couple, semblent être inhabituelles. ${ }^{9}$

Lorsqu'il s'agit de l'entrée en scène d'un nouveau couple, c'est-à-dire des nouveaux partenaires parentaux, les anciens partenaires sont consultés. C'est ainsi que Vincent et Victor, lorsque ce dernier a voulu s'engager dans une nouvelle coparentalité, ont d'abord demandé l'avis du couple de femmes avec lequel Vincent s'était engagé en premier. Un cadre éthique similaire apparaît même dans les cas où la coparentalité n'a pas pu se concrétiser. Avant que Paul, 45 ans, fonctionnaire, n'accepte de faire un enfant avec un couple d'amies, il a d'abord demandé à son ex-compagnon, qui s'était initialement engagé avec ce même couple avant de découvrir qu'il était stérile, s'ils ne s'opposait pas à ce qu'il le remplace. L'importance du respect de ces règles éthiques a été aussi soulignée par Eugène, même si, dans son cas, elles n'ont pas été respectées. Un beau jour, il apprend par sa fille, alors âgée de 5 ans, que sa mère " avait un bébé dans son ventre ". La nouvelle l'a " choqué ", car selon lui, la mère de sa fille ne lui avait pas demandé son avis. Comme il avait déjà des conflits avec elle, cette nouvelle coparentalité n’a fait que les réalimenter. Ces quelques exemples montrent que, dans un contexte marqué par la valorisation du " choix individuel ", la volonté des individus est limitée par un cadre éthique particulier créé à partir des rapports unissant les coparents.

9 La situation de Gabrielle ne figure pas sur le diagramme présenté précédemment. Hétérosexuelle, Gabrielle a proposé à un couple d'amis d'avoir un enfant avec chacun d'eux. 


\section{Devenir père dans le contexte de la coparentalité}

Dans les familles recomposées, situation qui semble la plus similaire à la coparentalité, le père et la mère ne vivant plus en couple, il faut réfléchir aux places qu'occuperont leurs nouveaux partenaires respectifs. Dans son analyse des figures paternelles présentes dans les familles recomposées, Agnès Martial (1998) démontre qu'avec l'augmentation des divorces, la paternité s'est émancipée du contexte institutionnel où elle était inscrite. Dans ce cas, le lien biologique a pris une importance croissante pour déterminer le « vrai » père de l'enfant. Parallèlement, la dimension « sociale " de la paternité, c'est-à-dire le partage du quotidien de l'enfant, est de plus en plus valorisée. La nouvelle norme de pérennité de liens parentaux fait du beau-père un parent additionnel, dans la mesure où il n'est pas censé se substituer au père. L'ancien et le nouveau compagnon de la mère peuvent alors se trouver dans une situation de concurrence.

Dans les coparentalités, le père et son compagnon ne sont pas dans une même relation de concurrence. Dans les situations où l'enfant a été un projet du couple, le parent sans statut légal fait partie de la vie de l'enfant dès sa naissance, contrairement à la situation vécue par le beau-père dans les familles recomposées. A première vue, la logique de substitution qui peut exister entre personnages familiaux de même sexe, et que l'on voit à l'œuvre dans certaines situations de recomposition, n'aurait pas lieu d'être dans une coparentalité. Et pourtant, l'idée d'un éventuel " partage » de la paternité a été clairement refusée par certains pères en coparentalité. Plutôt que d'imaginer un dédoublement de la position de père, ils la considèrent comme une place à usage unique.

Certains pères ont affirmé explicitement qu'ils voulaient avoir un enfant " de leur propre chair ». Pour eux, l'existence d'un « lien biologique » était une variable nécessaire, certes, mais pas suffisante pour l'établissement de leur paternité. L'engagement affectif auprès de l'enfant, le besoin ressenti d'être près de lui et de lui exprimer leur amour, définissent tout autant le contenu qu'ils veulent donner à leur rôle de pères. Or, leur réticence à partager la paternité n'a de sens que face à la possibilité concrète d'un tel partage. Le « lien biologique " est valorisé, entre autres raisons, parce qu'il donne un ancrage à la dimension sociale et affective de la paternité, qu'ils veulent exercer de manière exclusive. La possibilité d'un lien électif, tant valorisé par le discours militant de l'A.P.G.L., est ici, paradoxalement, ce qui expliquerait la 
résistance de certains pères à laisser une place parentale à leur compagnon.

Même dans les cas où les pères n'étaient pas hostiles à un " partage " de leur paternité, le lien « biologique » et légal liant un seul des deux à l'enfant transforme le statut et l'identité de son détenteur, créant ainsi une asymétrie entre les conjoints. Mais, au lieu de nier l'importance du « lien biologique », certains compagnons deviennent eux aussi des " pères biologiques ", ce qui permet de retrouver une symétrie des positions de chacun à l'intérieur du couple et de la famille. Virginie Descoutures (2005) décrit des pratiques semblables mises en œuvre par des couples lesbiens, qui, pour faire face à l'inégalité des statuts parentaux, d'autant plus ressentie que l'enfant est le résultat d'un projet de couple, cherchent un « équilibre » en se tournant parallèlement vers la conception. Les deux partenaires sont ainsi, « séparément mais réciproquement ", légalement reconnues comme parents.

Contrairement aux hommes qui font appel à des mères de substitution ou à l'adoption, ceux qui sont en coparentalité doivent partager l'enfant avec une femme, la mère, qui peut elle aussi être en couple. Or, dans les situations rencontrées, il y a des questions d'organisation entre les foyers qu'il faut gérer. La plupart de ce travail est réalisé par une seule personne du couple, le parent légal. Vincent dit, par exemple, que si d'un côté chacun peut s'occuper de l'enfant de l'autre quand ils sont à la maison, l'« interface » ou la " gestion des filles » est toujours faite par la même personne, le parent légal. Selon lui, c'est une façon de " partager le travail ".

Parallèlement, ce qui ressort des entretiens avec les pères en coparentalité, et qui apparaît moins dans les entretiens avec les autres pères, c'est la conscience d'une fragilité du lien paternel. Quand j’ai rencontré Julien, 40 ans, graphiste, la femme avec qui il allait avoir un enfant était enceinte. Ce moment était pour lui fortement chargé d'angoisse parce qu'il considérait que la grossesse, le fait de porter un enfant, créait déjà un lien entre la mère et son enfant alors que lui-même ne pourrait établir un lien qu'après la naissance. Le fait de toucher le ventre et de parler avec le bébé lui permettait déjà une forme de rapprochement avec l'enfant, dans la mesure où il croyait que l'enfant " allait s'habituer à sa voix ». En tout cas, il avait très peur qu'après l'accouchement la mère décide de s'en aller avec l'enfant. Même si, ici, cette peur de perdre l'enfant s'est révélée infondée, j'ai rencontré des situations où les pères ont été effectivement confrontés à une coupure du lien avec l'enfant. Ainsi, si ces pères ont du mal à partager leur place, c'est en partie 
parce que celle-ci n'est pas aussi assurée que celle de la mère. Comme la position de cette dernière est plus légitimée, le père et la compagne de celle-ci se trouvent parfois dans une situation de concurrence pour savoir lequel occupera la place de " deuxième " auprès de l'enfant (Gross 2006). Dans ce contexte, le compagnon du père apparaît comme le moins légitime à revendiquer une position parentale.

Les pères rencontrés réactualisent une conception de la paternité qui s'appuie sur la notion de " reconnaissance ", le « biologique » ou le " génétique " n'étant que l'un des supports possibles pour la " construction » du lien paternel (voir Schneider 1984). Ce lien n'est pas pensé comme une conséquence directe du partage d'une même substance, mais comme le résultat d'une " construction " qui passe par l'expérience, autrement dit par l'exercice d'un rôle. La maternité, au contraire, n'est pas perçue comme le fruit d'une " construction sociale » ou, en tout cas, pas complètement. Même ceux qui font preuve d'un regard critique à ce sujet n'endossent pas aisément une vision radicalement constructiviste de la maternité. Dans d'autres situations, le lien à la mère est plutôt pensé comme devant être " défait ", que ce soit par référence à la notion d' " abandon ", dans le cas de l'adoption, ou de " don altruiste ", dans le contexte de la maternité de substitution.

Cette question est importante car, même si la coparentalité peut paraître une modalité de création de liens familiaux mieux « adaptée » au monde moderne, où les dimensions de la conjugalité et de la parentalité ne sont plus liées de manière indissociable à l'institution du mariage, sa mise en pratique donne souvent lieu à des disputes entre les coparents, notamment entre hommes et femmes, pour savoir à qui appartient l'enfant. Des attentes divergentes sur le rôle et les prérogatives de chacun créent ainsi des situations de tension qui peuvent compromettre cet idéal d'harmonie, illusoirement alimenté par le contraste avec les familles recomposées issues d'une situation de divorce. Ces tensions peuvent être liées à des définitions divergentes sur la maternité et la paternité, ce qui peut causer des disputes sur le plan des représentations de genre.

\section{Considérations finales}

Les familles homoparentales posent des questions centrales dans le contexte contemporain de mutation des relations familiales. La coparentalité, quant à 
elle, remet en cause le principe d'exclusivité qui prévaut dans les définitions juridiques de la filiation. Les positions parentales se multiplient sans que des statuts puissent leur être clairement associés. Les rapports entre hommes et femmes se trouvent également problématisés, comme dans les situations nouvelles de procréation entre gays et lesbiennes, où la question de l'appartenance de l'enfant est sans cesse questionnée.

Dans plusieurs pays occidentaux, l'un des enjeux le plus important concernant les familles homoparentales n'est pas seulement celui de leur légitimité en tant que famille, mais aussi et surtout celui des modes juridiques de leur reconnaissance. De nouveaux dispositifs juridiques doivent être inventés pour officialiser les liens multiples produits par la coparentalité, qui gardent des spécificités importantes par rapport aux statuts des beaux-parents dans les familles recomposées. D’une manière inédite, les familles homoparentales mettent en perspective les fictions juridiques basées sur l'hétérosexualité et la procréation (Rubin 1975; Butler 2002). Il ne s'agit pas ici de la confrontation entre une définition "biologique » et une définition " sociale " de la filiation, mais de la pertinence, dans certaines configurations familiales contemporaines, de considérer la procréation comme un " modèle » pour l'établissement de la filiation. David Schneider $(1980 ; 1984)$ a déjà démontré combien l'opposition nature/loi est constitutive des représentations euroaméricaines sur la parenté. La question fondamentale n'est donc pas de savoir si c'est la " dimension sociale " ou la " dimension biologique " qui détermine la parenté, mais comment ces catégories sont signifiées et articulées dans un contexte particulier à partir de définitions spécifiques de la parenté (Fine 2006; voir aussi Viveiros de Castro 2004). Plus spécifiquement, les différentes configurations homoparentales questionnent la pertinence d'un modèle généalogique comme référence obligatoire pour la constitution de statuts de parenté. La littérature anthropologique a également démontré combien ce modèle est culturellement spécifique et non nécessaire (Bamford \& Leach 2009). Les familles homoparentales ont augmenté les références ethnographiques permettant de relativiser la nécessité d'un modèle généalogique basé sur la procréation et structuré sur l'exclusivité de positions et la différence sexuée. Leur compréhension exige ainsi une reformulation des concepts et des modèles théoriques utilisés par les sciences sociales pour analyser la parenté dans les sociétés occidentales contemporaines. 


\section{Références bibliographiques}

BAMFORD, Sandra; LEACH, James (orgs.). 2009. Kinship and beyond. The genealogical model reconsidered. Oxford: Berghahn.

BOZON, Michel. 2002. Sociologie de la sexualité. Paris: Nathan.

BUTLER, Judith. 2002. "Is kinship always already heterosexual?". differences: A Journal of Feminist Cultural Studies, 13(1): 14-44.

CADORET, Anne. 2002. Des parents comme les autres - homosexualité et parenté. Paris: Éditions Odile Jacob.

DE SINGLY, François. 2004. Le soi, le couple et la famille. Paris: Nathan.

DESCOUTURES, Virginie. 2005. "Le travail d'institution de la famille homoparentale. Entre droit à la différence et droit à l'indifférence”. In: M. Gross (org.), Homoparentalités. État des lieux. Paris: Érès. pp. 345-355. DESCOUTURES, Virginie. 2008. Les mères lesbiennes. Contribution à une sociologie de la parentalité. Thèse de doctorat en sociologie, Université Paris Descartes - Paris.

FASSIN, Éric. 2005. “Couples homosexuels et familles homoparentales”. In: M. Maruani (org.), Femmes, sexe ou genre. L'état des savoirs. Paris: La Découverte. pp. 184-191.

FESTY, Patrick. 2006. "Le recensement des familles homoparentales”. In: A. Cadoret; M. Gross; C. Mécary; B. Perreau (orgs.), Homoparentalités. Approches scientifiques et politiques. Paris: PUF. pp. 109-116.

FINE, Agnès. 2001. "Pluriparentalité et système de filiation dans les sociétés occidentales”. In: D. Le Gall; Y. Bettahar (orgs.), La pluriparentalité. Paris: PUF. pp. 69-93.

FINE, Agnès. 2006. "Pluriparentalités et homoparentalités dans les sociétés occidentales contemporaines”. In: A. Cadoret; M. Gross; C. Mécary; B. Perreau (orgs.), Homoparentalités. Approches scientifiques et politiques. Paris: PUF. pp. 43-55.

FOUCAULT, Michel. 1994. Histoire de la sexualité - la volonté de savoir. Paris: Gallimard.

GODELIER, Maurice. 2004. Métamorphoses de la parenté. Paris: Fayard.

GRATTON, Emmanuel. 2008. L'homoparentalité au masculin. Paris: Le Monde/ PUF.

GROSS, Martine; PEYCERÉ, Mathieu. 2005. Fonder une famille homoparentale. Paris: Ramsay.

GROSS, Martine (org.). 2005. Homoparentalités. État des lieux. Paris: Érès. 
GROSS, Martine. 2006. "Désir d'enfant chez les gays et les lesbiennes”. Terrain, 46: 151-164.

MARTIAL, Agnès. 1998. "Pères et beau pères. Figures paternelles et recompositions familiales”. In: A. Fine; C. Laterrasse; C. ZaoucheGaudron (orgs.), A chacun sa famille: approche pluridisciplinaire. Toulouse: Éditions Universitaires du Sud. pp. 81-94.

MEHL, Dominique. 2003. La bonne parole, quand les psys parlent dans les media. Paris: Ed. de la Martinière.

OUELLETTE, Françoise-Romaine. 1998. "Les usages contemporains de l'adoption". In: A. Fine (org.), Adoptions. Ethnologie des parentés choisies. Paris: Éditions de la Maison des Sciences de l'Homme. pp. 153-176. OUELLETTE, Françoise-Romaine. 2000. "L’adoption face aux définitions de la famille et de l'institution généalogique”. In: A. Fine; C. Neirinck (orgs.), Parents de sang, parents adoptifs. Approches juridiques et anthropologiques de l'adoption. France, USA, Canada, Europe. Paris: LGDJ. pp. 325-341.

PEERBAYE, Ashveen. 200o. L'invention de l'homoparentalité - Acteurs, arènes et rhétoriques autour de la question de la filiation homosexuelle. Mémoire de DEA en Sciences Sociales, ENS, Cachan.

RUBIN, Gayle. 1975. "The traffic in women: notes on the 'political economy' of sex". In: R. Reiter (org.), Toward an anthropology of women. New York/London: Monthly Review Press. pp. 157-210.

SCHNEIDER, David M. 1980. American kinship. Chicago/London: University of Chicago Press.

SCHNEIDER, David M. 1984. A critique of the study of kinship. Ann Arbor: University of Michigan Press.

TARNOVSKI, Flávio Luiz. 2002. "Pais assumidos": adoção e paternidade homossexual no Brasil contemporâneo. Mémoire de " Mestrado " en Anthropologie Sociale, UFSC - Florianópolis.

TARNOVSKI, Flávio Luiz. 2010. Être père et homosexuel dans la France contemporaine. Thèse de doctorat en Anthropologie Sociale, EHESS Toulouse.

THÉRY, Irène. 1991. "Trouver le mot juste: langage et parenté dans les recompositions familiales après divorce”. In: M. Segalen (org.), Jeux de familles. Paris: Presses du CNRS. pp. 137-156.

THÉRY, Irène. 1996. "Différence des sexes et différence des générations. L'institution familiale en déshérence”. Esprit, 227: 65-90. 
THÉRY, Irène. 1998. Couple, filiation et parenté aujourd'hui. Paris: Odile Jacob. VIVEIROS DE CASTRO, Eduardo. 2004. "Le don et le donné: trois nano-essais sur la parenté et la magie”. Ethnographiques.org, n. 6. [En ligne: www. ethnographiques.org/Numero-6-novembre-2004.html, consulté le: 31.03.2008].

\section{A propos de l'auteur}

Flávio Luiz Tarnovski est docteur en anthropologie sociale et ethnologie, diplômé de l'École des hautes études en sciences sociales. Sa thèse, soutenue en 2010, porte sur les familles homoparentales dans la France contemporaine. Membre associé du L.I.S.S.T./Centre d'Anthropologie de Toulouse, il est actuellement enseignant-chercheur (professor adjunto) à l'Université Fédérale de Mato Grosso, à Cuiabá/Brésil.

e-mail : flaviolt@gmail.com

Adresse:

Universidade Federal de Mato Grosso

Departamento de Antropologia

Av. Fernando Corrêa da Costa, 2367 - Boa Esperança

78060-90o Cuiabá/MT 